\title{
Article \\ A Switching-Based Control Method for the Fairing Separation Control of Axisymmetric Hypersonic Vehicles
}

\author{
Qin Zhong ${ }^{1, *}$, Yonghua Fan ${ }^{1}$ and Wenbin $\mathrm{Wu}^{2}$ \\ 1 School of Astronautics, Northwestern Polytechnical University, Xi'an 710072, China; fyhlixin@163.com \\ 2 Institute of Modern Control Technology, Xi'an 710065, China; anson616.ok@163.com \\ * Correspondence: qzhong@mail.nwpu.edu.cn; Tel.: +86-13572082215
}

Citation: Zhong, Q.; Fan, Y.; Wu, W. A Switching-Based Control Method for the Fairing Separation Control of Axisymmetric Hypersonic Vehicles. Aerospace 2022, 9, 132. https:// doi.org/10.3390/aerospace 9030132

Academic Editor: Roberta Fusaro

Received: 15 January 2022

Accepted: 1 March 2022

Published: 3 March 2022

Publisher's Note: MDPI stays neutral with regard to jurisdictional claims in published maps and institutional affiliations.

Copyright: () 2022 by the authors. Licensee MDPI, Basel, Switzerland. This article is an open access article distributed under the terms and conditions of the Creative Commons Attribution (CC BY) license (https:// creativecommons.org/licenses/by/ $4.0 /)$.

\begin{abstract}
For hypersonic-vehicle fairing separation, the reliable separation of the fairing and rapid suppression of the separation disturbance are required in scenarios where there is high dynamic pressure and there are abrupt changes in the aerodynamic shape of the aircraft as well as overall parameters. A switching-based control method is proposed in this paper for the interference suppression of hypersonic-vehicle fairing separation, aiming to ensure the stability of the control system in cases of abrupt changes in the controlled object. First, an unsteady-flow calculation method is adopted to clarify the aerodynamic interference characteristics of the aircraft in fairing separation, and the aerodynamic calculation model of the hypersonic-vehicle fairing-separation process is established; then, two states of the aircraft, with and without fairing, are transformed into a subsystem with switching characteristics. The attitude-stability problem in the fairing-separation process is transformed into a problem of solving the arbitrary switching of the switched system by using the linear matrix inequality (LMI) approach. Meanwhile, the control system for interference suppression of the fairing separation is designed by considering the aerodynamic interaction of the fairing movement with the aircraft during the separation. The simulation results showed that the designed control system can realize the reliable separation of the fairing under high dynamic pressure and effectively suppress interference in the separation process.
\end{abstract}

Keywords: axisymmetric; hypersonic vehicle; fairing separation; switch control; separation disturbance

\section{Introduction}

The typical separation flight altitude of a near-space air-breathing hypersonic vehicle is $20-40 \mathrm{~km}$, with a flight speed of Mach 5-7, and a separation dynamic pressure of 50-170 Kpa. Only in this way can the scramjet engine be reliably ignited. Whether launching in the air or from the ground, there is a process of accelerating from a low speed to a suitable speed for ignition, with the dynamic pressure varying from tens to hundreds of kilopascals during the flight. Before ignition of the scramjet engine, the inlet flies under a high dynamic pressure for a long time, which can easily damage the internal flowpath of the engine. Therefore, a process of fairing separation occurs before ignition of the scramjet engine. For example, the X-43A aircraft adopts a small hood that only protects the inlet, whereas the HyFly drone uses an integral bow cap to completely cover the head of the aircraft. Whether covered by a small hood or an integral bow cap, hypersonic vehicles always undergo fairing separation at high speed and under high dynamic pressure. In addition, because the bow cap is in front of the aircraft fuselage, the fairing movement will cause strong airflow interference with the aircraft, which poses a severe challenge to the attitude stabilization of hypersonic vehicles.

Several studies [1-5] have analyzed the feasibility of X-43A interstage separation and the aerodynamic characteristics of separation. Yang et al. [6] elaborated on the interstageseparation strategy and flight-test verification of the X-43A hypersonic vehicle. Using the linear-interpolation method, Li et al. [7] studied the aerodynamic characteristics of the 
interstage separation and the interstage-separation problem of the X-43A. In summary, current research on the high dynamic-pressure-separation control of hypersonic vehicles mainly focuses on the interstage separation of the booster and the aircraft. Furthermore, the interstage-separation control strategy mostly adopts the method of control augmentation; however, few studies have explored the control of inlet fairing separation.

Compared with interstage separation, fairing separation is complex in its aerodynamic and interference characteristics, mainly reflected in the following: (1) the general parameters and shape of the hypersonic vehicle will not change in the process of interstage separation, but they often greatly change during the fairing-separation process. (2) when interstage separation occurs, the hypersonic vehicle is in front of the booster so the separation disturbance has less impact on the vehicle, whereas in fairing separation, the vehicle is behind the fairing, and the shockwave interference caused by the fairing movement will act on the body of the vehicle. Therefore, in the process of fairing separation, the hypersonic vehicle will be subject to the following: impulse interference of the explosive bolt, abrupt changes in the aerodynamic shape of the vehicle itself and the overall parameters after the fairing separation, and flow-field interference caused by the forward and lateral motions of the fairing.

In the fairing-separation process, the hypersonic vehicle, as a control object, changes from a state with fairing to a state without fairing; then, the aerodynamic shape and overall parameters of the aircraft will undergo larger, more abrupt changes. Therefore, the aircraft before and after fairing separation can be regarded as two subsystems with independent features. Compared with other commonly used control methods, switching control can effectively solve the robust-control problem among multiple subsystems. Yang [8] proposed an arbitrary switching control technique based on pole assignment in the circle. The variational method was used in [9] to study the system stability of a linear switching system with two subsystems under the action of arbitrary switching signals. Another study [10] proposed a method following the general Lyapunov function, and presented the condition of global uniform asymptotic stability of the switching system (i.e., the system is asymptotically stable for any switching signal). In other words, there is a general Lyapunov function in the switching system.

For a hypersonic vehicle with fairing separation, its separation process has characteristics such as strong aerodynamic interference, abrupt changes in controlled objects, and a discontinuous control-system status. In the traditional control method, the control system for states with and without fairing is designed to achieve direct switching, which can easily cause divergence of the system. Therefore, in view of the above problems, this paper proposes a switching-based control method for hypersonic-vehicle fairing separation. According to the aerodynamic characteristics of the hypersonic vehicle, the method regards the two states of the aircraft with and without fairing as two subsystems of the switching system, and transforms the attitude-stability problem in fairing separation into the arbitrary switching of two independent subsystems.

Moreover, the aerodynamic interaction of the fairing movement on the aircraft during separation is considered, and a control system for interference suppression of the fairing separation is designed. Simulation results show that the designed control system can realize reliable separation of the fairing under high dynamic pressure and achieve effective suppression of the interference in the separation process.

\section{Materials and Methods}

\subsection{Description of the Hypersonic-Vehicle Fairing Separation Process}

The research object of this paper is a hypersonic vehicle with an integral hood, based on an actual engineering project; an outline diagram is presented in Figure 1. The aircraft features an axisymmetric shape, with an integral hood at the front. 


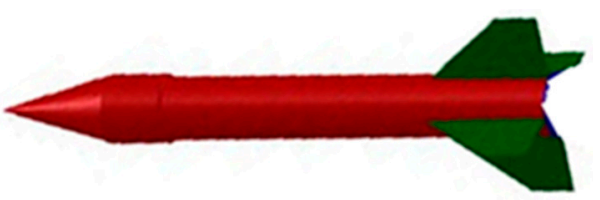

Figure 1. Hypersonic vehicle in the state with fairing.

Fairing separation adopts integral forward pushing and side pushing. The initial velocity of the hood at separation is $5.6 \mathrm{Ma}$. The separation process is as follows:

$t_{0}$ : A command is sent to separate the fairing. The explosive bolt detonates and the fairing disconnects from the aircraft;

$t_{1} \sim t_{2}$ : The forward-pushing engine on the fairing ignites, which impels the fairing to move forward along the slideway until the cover body completely disengages from the aircraft;

$t_{2} \sim t_{3}$ : The fairing moves forward until it reaches a safe distance, and there is no airflow interference between the fairing and the aircraft. The aerodynamic interference of the fairing movement process on the aircraft changes from strong to weak;

$t_{3} \sim t_{4}$ : The fairing slideway pushes the engine to work. It moves laterally ahead of the aircraft to prevent a collision.

The fairing-separation process is shown in Figure 2.

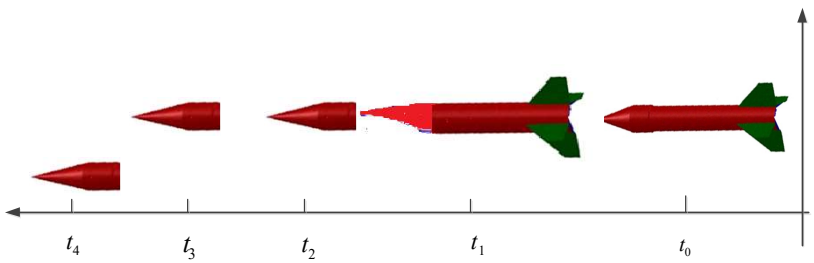

Figure 2. Schematic diagram of the fairing-separation process.

In the process of fairing separation in hypersonic vehicles, there is a very complex aerodynamic-interference field. In this study, the trajectory and force of the hypersonicvehicle fairing-separation process were simulated by a CFD method based on overlappinggrid technology. The CFD tool used in this paper is developed by China Aerodynamics Research and Development Center. The software can simulate the application scenarios of aircraft, helicopters, missiles, reentry vehicles, and other aircraft with low speed, subsonic, transonic, supersonic, and hypersonic speed, such as steady and unsteady aerodynamic forces, multibody motion, inlet internal flow, jet-flow field, and so on. The software is characterized by multibody motion simulation and covers typical CFD processes such as grid processing, numerical solution, and engineering data processing.

The calculation process was:

a. Generating the initial grid, including the background grid of the aircraft with booster stage before package separation and the subgrid of the aircraft;

b. Automatic hole digging: perform automatic hole digging on the background grid and subgrid, judge whether the grid is in the calculation area, and build the overlapping relationship between them so as to establish the interpolation data structure in the calculation process;

c. Flow-field solution: for the established overlapping grid, the flow-field solver is used to calculate and obtain the convergent aerodynamic characteristics;

d. Solution to the 6DOF equation: calculate the velocity, position, and attitude by using the calculated aerodynamic force and torque;

e. According to the obtained speed, position, and attitude, the mesh is moved and rotated. At the same time, judge whether the separated object collides with the carrier. If it collides, exit the calculation;

f. Repeat steps b to e until the aircraft is separated to a safe distance. 
The numerical simulation method for analyzing aircraft aerodynamic characteristics mainly includes unsteady calculation, control equation, discretization, and boundary conditions. The unsteady method is used to calculate the aerodynamic interference in the separation process of hypersonic vehicles, and its control equation is:

$$
\frac{\partial}{\partial t} \iiint_{V} Q d V+\oiiint_{\partial \Omega}\left(H(Q) \cdot \hat{n}-Q \vec{v}_{g} \cdot \hat{n}\right) d S=\oiiint_{\partial \Omega} H_{v}(Q) \cdot \hat{n} d S
$$

where $Q=\left[\begin{array}{lllll}\rho & \rho u & \rho v & \rho w & \rho E\end{array}\right]^{T}$ is the conserved variable vector, $H$ is the inviscid flux vector, $H_{v}$ is the viscous flux vector, $v_{g}$ is the velocity of the face $d S$, and $\hat{n}$ is the normal vector of the face $d S$.

The governing equation in the unit body is obtained by integrating the equation:

$$
\frac{\partial(Q V)}{\partial t}+\sum_{k=1}^{N}\left(H_{k} \cdot \hat{n}_{k}-Q \vec{v}_{g k} \cdot \hat{n}_{k}\right) S_{k}=\sum_{k=1}^{N} H_{v k} \cdot \hat{n}_{k} S_{k}
$$

where $V$ is the unit volume, $\hat{n}_{k}$ is the unit external normal vector of the unit surface, $N$ is the total number of surfaces of the unit $(k=1,2, \ldots, N), S_{k}$ is the area of the surface $k$, and $H_{k}$ and $H_{v k}$ are the normal inviscid and viscous flux of the surface $k$.

The velocity $v_{n k}=\overrightarrow{v_{g k}} \cdot \hat{n}_{k}$ of the element surface $S_{k}$ can be obtained directly from the coordinates of the face-center coordinates in the time layer $n+1$ and the time layer $n$, which is $v_{n k}=\left|x_{s c}^{n+1}-\overrightarrow{x_{s c}^{n}}\right| \cdot \hat{n}_{k} / \Delta t$.

Let $R(Q)=\sum_{k=1}^{N}\left(H_{k} \cdot \hat{n}_{k}-Q \vec{v}_{g k} \cdot \hat{n}_{k}\right) S_{k}-\sum_{k=1}^{N} H_{v k} \cdot \hat{n}_{k} S_{k}$; then

$$
\frac{(1+\phi)\left(Q^{n+1} V^{n+1}-Q^{n} V^{n}\right)-\phi\left(Q^{n} V^{n}-Q^{n-1} V^{n-1}\right)}{\Delta t}+R\left(Q^{n+1}\right)=0
$$

where $\phi=0$ is the first-order accuracy of time and $\phi=1 / 2$ is the second-order accuracy of time. Double time stepping, also known as pseudo-time sub-iteration, $\tau-T S$, is used to solve the equation. Thus, the virtual time step term $\frac{\partial(Q V)}{\partial \tau}$ is directly added to the equation, and the equation becomes:

$$
\frac{\partial(Q V)}{\partial \tau}+\frac{(1+\phi)\left(Q^{n+1} V^{n+1}-Q^{n} V^{n}\right)-\phi\left(Q^{n} V^{n}-Q^{n-1} V^{n-1}\right)}{\Delta t}+R(Q)=0
$$

The steady solution of the equation is obtained by the step-by-step solution of the virtual time step $\Delta \tau$ is $Q^{n+1}$; then, it can be used to continue the solution of the next time step $Q^{n+1}$.

For the steady solution of the equation, the LU-SGS method is adopted for time discretization, and the following can be obtained:

$$
\begin{aligned}
& \left(\frac{V_{i}^{n+1}}{\Delta \tau}+\frac{(1+\phi) V_{i}^{n+1}}{\Delta t}+\frac{1}{2} \sum_{k=1}^{N} \tilde{\lambda}_{\max } S_{k}\right) \delta Q_{i}{ }^{*} \\
& =-R H S\left(Q_{i}^{m}\right)-\frac{1}{2} \sum_{k=1}^{N}\left[\left(H\left(Q_{j}^{m}+\delta Q_{j}^{*}\right)-H\left(Q_{j}^{m}\right)\right)-\lambda_{\max } \delta Q_{j}^{*}\right] S_{k} \\
& \quad \delta Q_{i}^{m+1}=\delta Q_{i}^{*}-\frac{1}{2} \cdot\left(\frac{V_{i}^{n+1}}{\Delta \tau}+\frac{(1+\phi) V_{i}^{n+1}}{\Delta t}+\frac{1}{2} \sum_{k=1}^{N} \widetilde{\lambda}_{\max } S_{k}\right)^{-1} \\
& \quad \times \sum_{k=1}^{N}\left[\left(H\left(Q_{j}^{m}+\delta Q_{j}^{m+1}\right)-H\left(Q_{j}^{m}\right)\right)-\lambda_{\max } \delta Q_{j}^{m+1}\right] S_{k}
\end{aligned}
$$


where $\widetilde{\lambda}_{\text {max }}$ is the spectral radius $\widetilde{\lambda}_{\max }=\lambda_{\max }-v_{n k}$

$$
\begin{aligned}
& -\operatorname{RHS}\left(Q_{i}^{m}\right)=-\frac{(1+\phi) Q_{i}^{m} V^{n+1}}{\Delta t}+\frac{(1+\phi) Q_{i}^{n} V^{n}+\phi\left(Q_{i}^{n} V^{n}-Q_{i}^{n-1} V^{n-1}\right)}{\Delta t} \\
& -\sum_{k=1}^{N} \widetilde{H}_{k}\left(Q^{m}\right) \cdot \hat{n}_{k} S_{k}+\sum_{k=1}^{N} H_{v k}\left(Q^{m}\right) \cdot \hat{n}_{k} S_{k}
\end{aligned}
$$

The roe flux-differential splitting method is used for inviscid flux, Venkat is used for the limiter, and the center format is used for viscous term. Due to the long work cycle of unsteady calculations, local time steps and multigrid techniques are also used in the subiteration to improve the calculation efficiency. The turbulence model is a oneequation SA model. The multigrid technology used in this paper is based the "directcutting" strategy. This method does not need assistant mesh and has excellent robustness. The core algorithm of the multigrid technology includes overlapping assembling and overlapping interpolation. The purpose of overlapping assembling is to define the attributes of mesh elements, including active elements, interpolation elements, and inactive elements. Overlapping interpolation needs to search for the contributing unit corresponding to the unit to be interpolated, then transfer the flow field between grids through the trilinear interpolation method. The ADT method is used while searching for the contribution unit to accelerate the algorithm. The grid moves synchronously with the separation body, which is divided into translation and rotation. The translation part is coordinate displacement, and the rotation part constructs the rotation matrix through the attitude angle.

The main calculative parameters are as follows:

1. Mach number, 5.6; altitude, $21 \mathrm{~km}$; angle of attack, $5.0^{\circ}$; sideslip angle, $0.0^{\circ}$.

2. Courant-Friedrichs-Lewy (CFL) number, 0.8; number of steps, 2000; number of multigrid layers, 5;

The CFD calculation adopts high-performance computing-cluster 64-core parallel computing, the turbulence model adopts the SA equation, the spatial discrete format adopts the roe format, and the time advance adopts the implicit two-step time method.

The six-degrees-of-freedom calculation was conducted from $0.0 \mathrm{~s}$ to $0.24 \mathrm{~s}$ of the time node, and the grid was automatically divided every $20 \mathrm{~ms}$ to divide the fairing separation process into 12 stages. Therefore, after the relative displacement between the fairing and aircraft, the grid needs to be updated every $20 \mathrm{~ms}$ to adapt to the subsequent flow simulation. Moreover, the initial relative speed of the fairing to the aircraft is 0 . The number of steps, 2000, means that for each inner iteration the time for performing a steady calculation is about $6 \mathrm{~h}$, whereas the time for performing an unsteady calculation is about $65 \mathrm{~h}$. When the aerodynamic values tend to be stable, the results are considered to converge.

Overlapping-grid technology is key in hypersonic multibody separation simulations. In order to realize the accurate simulation of relative motion between multibodies, corresponding grids are generated for different objects, and the flow-field information is transmitted between different grids through interpolation technology. Overlapping-grid technology mainly includes overlapping hole excavation, contribution element search and flow field interpolation.

In this study, the "object-surface intersection" direct hole-digging method was adopted, which has high robustness and can easily be extended to dig holes between multiple sets of meshes. In terms of contribution-unit searches, at each time step, the alternative digital tree (ADT) method is used to find the corresponding relationship between all nodes of background grid A and subgrid B control units, and search for the contribution unit of the unit to be interpolated. This method arranges the spatial points in order and constructs them into binary tree data, which improves the efficiency of the query. In this study, the control volume was constructed as binary tree data to find the grid nodes in the control volume; trilinear interpolation was used to realize the data transmission between different grids. Trilinear interpolation is commonly used in the finite-element method. Unlike the distance-weight method which only considers the distance, trilinear interpolation regards the flow field as a continuous linear distribution in the contribution element, and performs 
a linear interpolation along three directions of space. Trilinear interpolation can ensure the continuity of the interpolation of the elements on both sides on the interface of the two elements, i.e., the values of the points on the interface are the same based on the interpolation of the elements on both sides, so as to avoid interpolation discontinuities near the interface [11]. Zhang et al. studied the aerodynamic characteristics of grid rudder by numerical simulation. Through the comparison of the aerodynamic characteristics of grids with different shapes, the design scheme with better aerodynamic characteristics and structural strength is given [12].

In order to solve the N-S equation numerically, the mesh generation must be carried out. In this study, the unstructured grid element mixed with triangular prism and tetrahedron was adopted, as was the idea of three-level grid generation. In the area close to the boundary layer of the object surface, the triangular prism-grid element with a layer structure was adopted to simulate its viscous effect, and the tetrahedral element in the spatial area near the object surface was properly densified to capture its spatial shock wave and vortex structure, The far-field area was filled with smooth-transition tetrahedral elements to meet the far-field requirements of flow-field simulation. The generated typical regional grid is shown in Figure 3. The total grid consisted of about 13 million elements, including about 5 million triangular prism elements and 8 million tetrahedral elements.

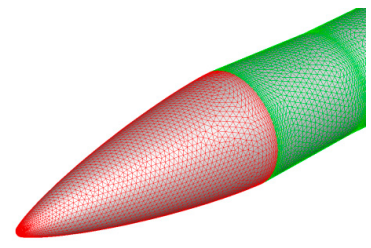

(a)

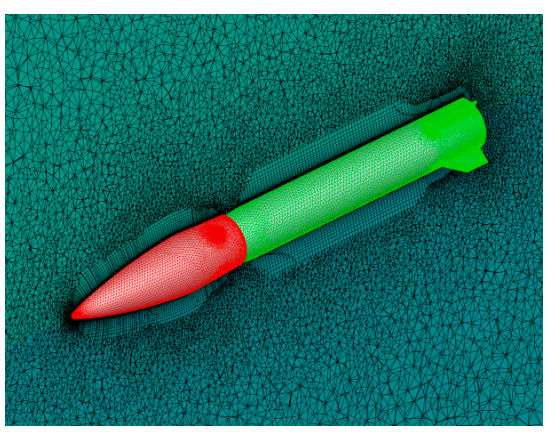

(c)

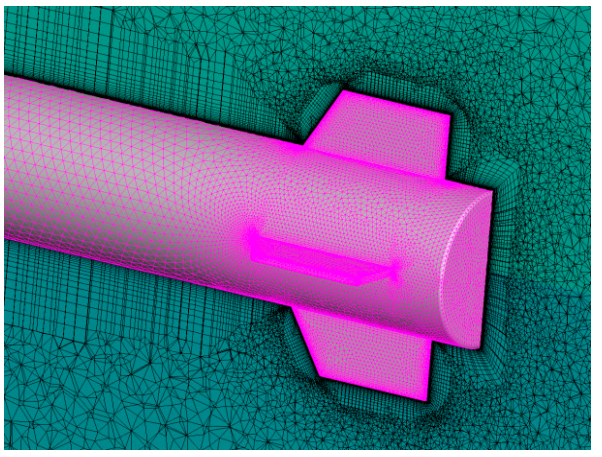

(e)

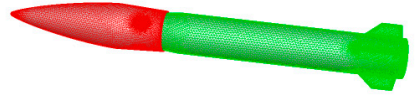

(b)

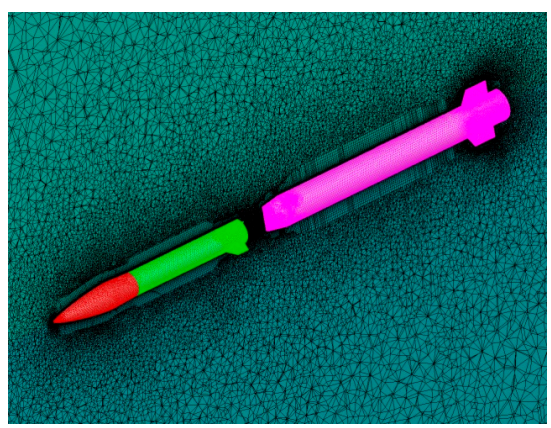

(d)

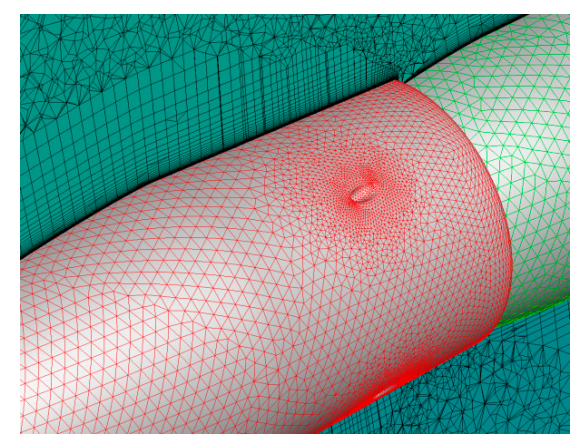

(f)

Figure 3. (a)Fairing-area grid; (b) rudder-wing grid; (c) boundary-layer mesh; (d) symmetrical-plane mesh; (e) the grid near the rudder surface; (f) the grid near the nozzle. 
Using the numerical-simulation method established above, aerodynamic-interference simulation of the hypersonic-vehicle fairing-separation process was carried out. The fairing separation was carried out through the fairing engine, which was simulated through the given jet conditions, considering the interference effect of the jet; the total pressure of the jet nozzle was $19 \mathrm{MPa}$ and the thrust was $10 \mathrm{kN}$.

Figure 4 shows the pressure-distribution cloud diagram of the symmetrical plane at different times during the fairing-separation process. It can be seen that during the separation process of the fairing, a strong detached oblique-shock-wave structure is generated at the front end of the hood, resulting in large aerodynamic resistance on the fairing hood. When the fairing is separated from the aircraft, shock waves will also be generated at the front end of the aircraft. However, this magnitude did not interfere with the flow field at first. When the fairing was completely separated from the aircraft, the shock interference at the front end of the aircraft began to become obvious. The forward thrust provided by the jet engine needs to overcome the resistance generated by the head shock wave; the high-pressure gas from the engine jet has a certain effect on the incoming flow, and an obvious separation-shock-wave structure is produced in front of the nozzle. With the increasing distance between the hood and the aircraft, the windward surface of the front end of the aircraft is gradually impacted by the high-pressure air flow, and the shock wave of the aircraft head is gradually enhanced. At around $0.12 \mathrm{~s}$, the rectifier hood and the missile body are mostly pulled out. At $0.22 \mathrm{~s}$, the axial distance between the hood and the aircraft has reached the length of the missile body of the aircraft. At this point, it is essentially safe to separate the head cover from the missile body.

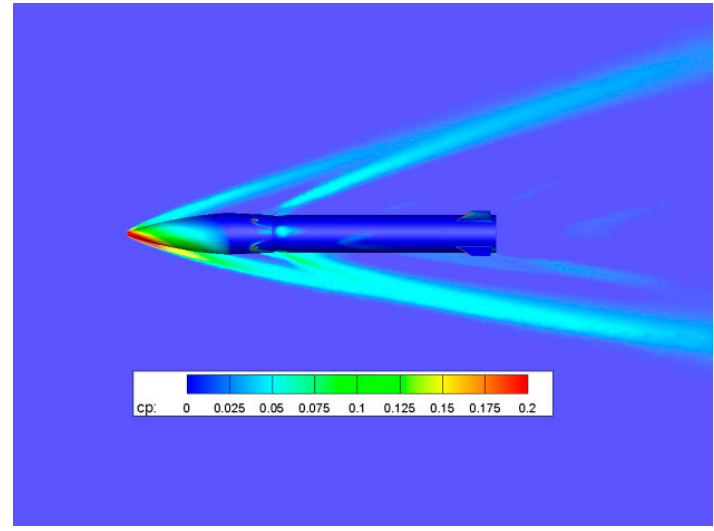

(a)

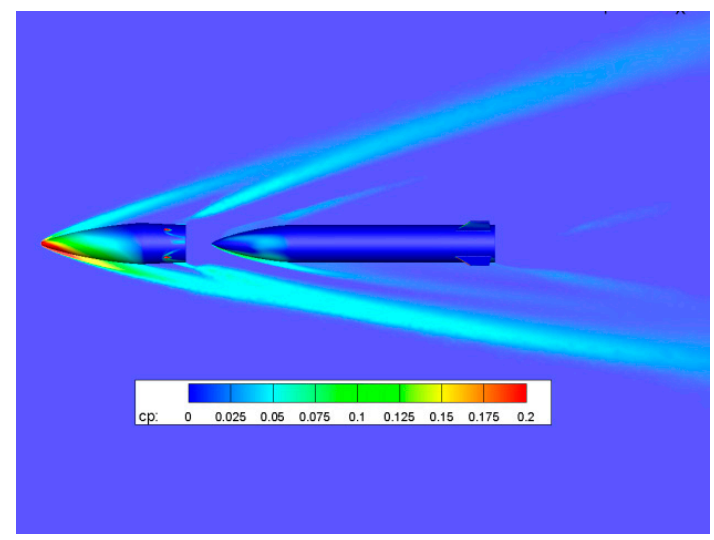

(c)

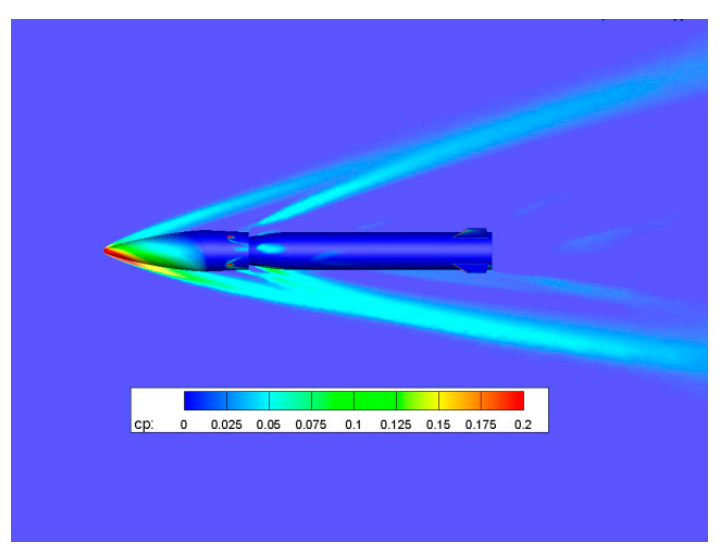

(b)

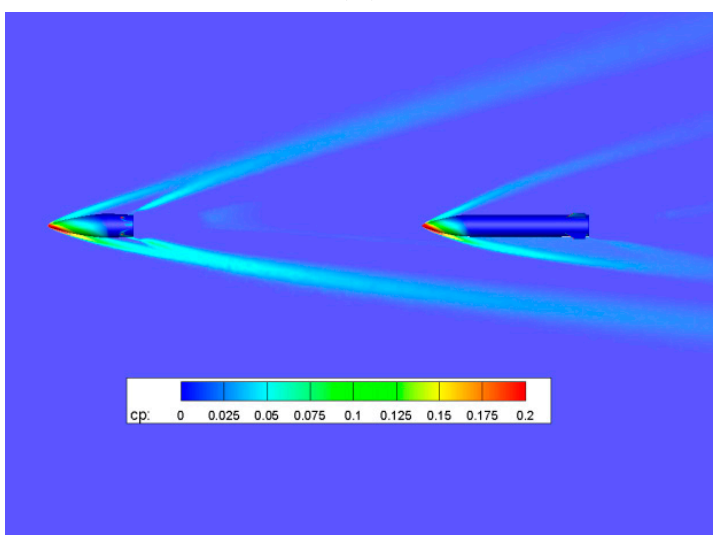

(d)

Figure 4. (a)Pressure contours of the fuselage at $0 \mathrm{~ms}$; (b) pressure contours of the fuselage at $80 \mathrm{~ms}$; (c) pressure contours of the fuselage at $120 \mathrm{~ms}$; (d) pressure contours of the fuselage at $220 \mathrm{~ms}$. 
Figure 5 shows the variation curves of the lift, drag, lateral force, and pitching moment coefficients of the verification machine and the fairing during the separation process.

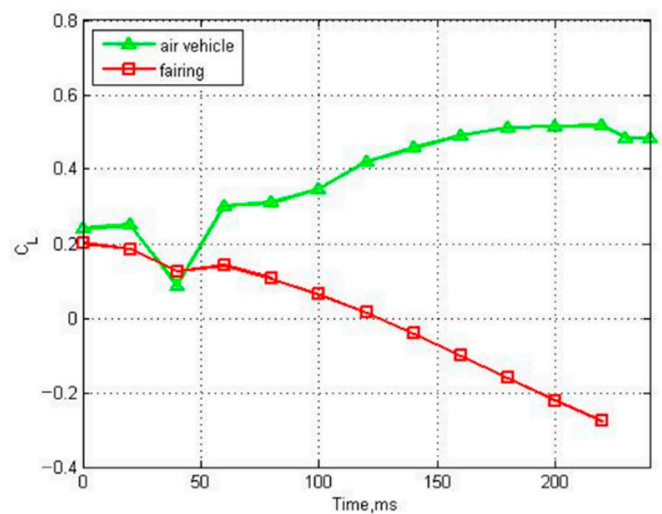

(a)

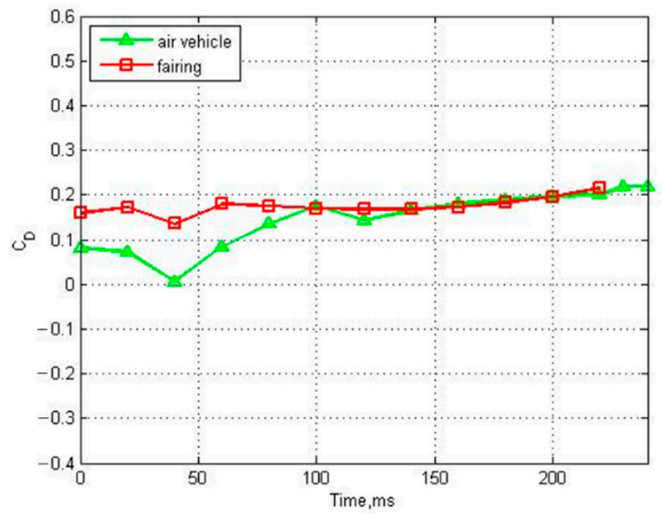

(c)

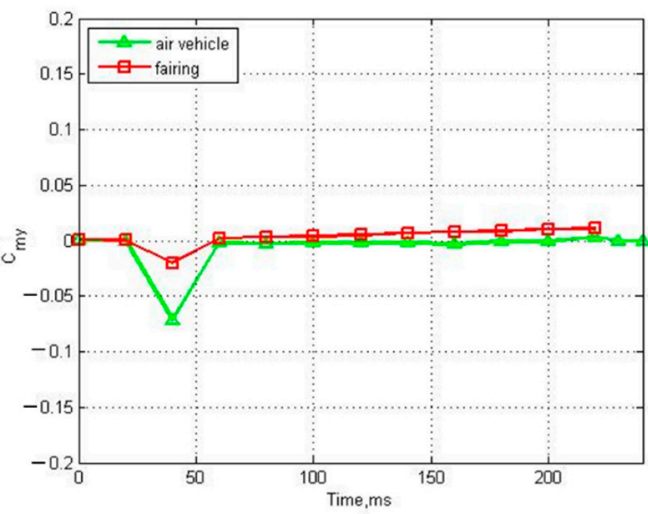

(b)

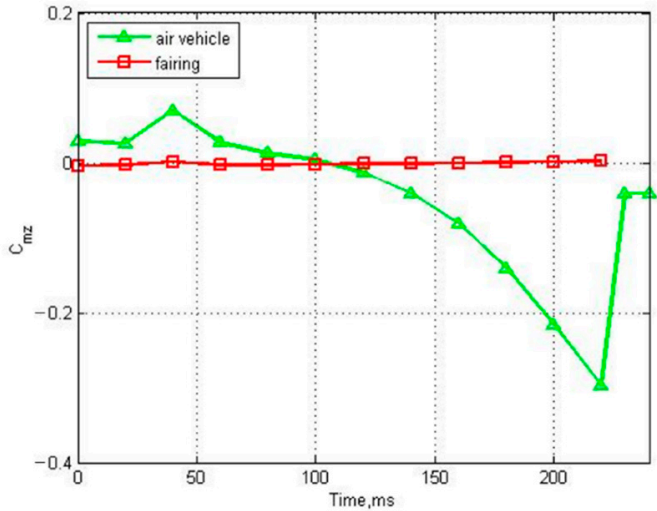

(d)

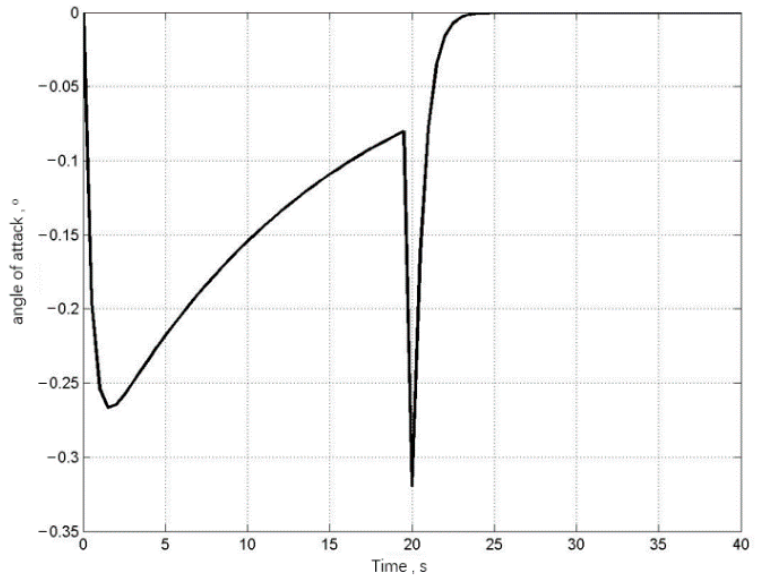

(e)

Figure 5. (a)Variation in the lift coefficient with time; (b) variation in the lateral force coefficient with time; (c) variation in the drag coefficient with time; (d) variation in the pitching moment coefficient with time; (e) angle of attack with interference.

It can clearly be seen from Figure 5 that the fairing has an obvious airflow interference with the aircraft during the separation process. Before the aircraft is completely separated from the fairing, the lift coefficient, drag coefficient, and lateral force coefficient spike, especially between 40 and $70 \mathrm{~ms}$. When the fairing has been separated from the body for a certain distance, affected by the interference of the high-pressure flow field generated by the fairing, the pitching moment coefficient of the aircraft decreases sharply to a negative value, 
which will exert an anti-manipulation effect on the control effect until the fairing is safely separated from the body. From the Figure $5 d$, the curve of the pitching moment coefficient $C_{m z}$ starts from a positive number when the separation began; along with the separation, the pitching moment coefficient declines to a negative value rapidly. The sign change of the pitching moment coefficient means that the control effect of the tail will also change; that is, if the control strategy before the sign change is continued, the opposite result will appear. From Figure 5e, it can be seen that the aerodynamic interference generated by fairing separation has a great impact on the angle of attack of the aircraft. The impact is adverse to the attitude stability of the aircraft.

\subsection{Dynamic Modeling of Hypersonic Vehicle}

According to the flow-field analysis in Section 2.1 of the fairing-separation stage of the hypersonic vehicle, the interference load experienced by the aircraft in the fairingseparation stage mainly includes the impact interference of the explosion bolt at the time of fairing separation. The sudden change in flow field is caused by the changes in aerodynamic shape and overall parameters of the hypersonic vehicle after the fairing is disengaged. Flow-field interference is caused by the forward and lateral movement of fairing. It is considered to decompose the above interference into three channels: roll, yaw, and pitch The specific form is shown in the Figure 6.
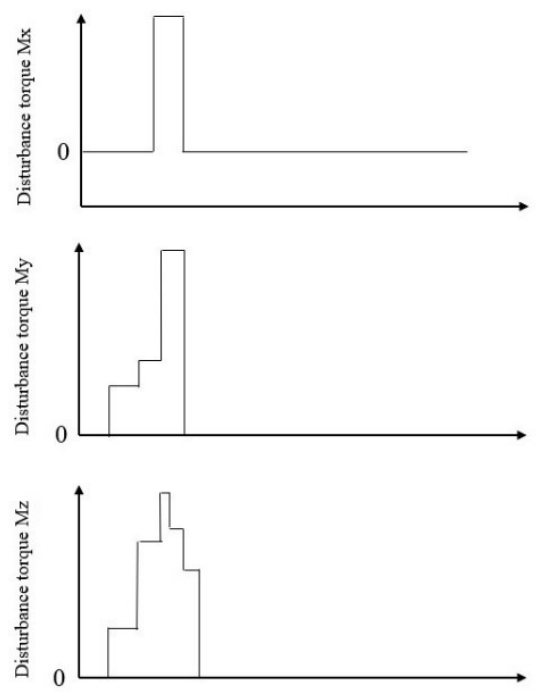

Figure 6. Schematic diagram of interference load in fairing separation.

Notably, in the process of fairing separation, interference of the body is present in the longitudinal and lateral channels: the impact interference of the explosion bolt mainly acts on the longitudinal channel; the shape parameters and flow-field interference act on the longitudinal channels at the same time; and the rolling channel is more or less the same as that of the general aircraft. Therefore, the longitudinal channel should mainly be considered in the controller design. The dynamic model of a hypersonic vehicle in the process of fairing separation is given below:

$$
\begin{aligned}
& m \frac{d V}{d t}=-X-m g \sin \theta \\
& J_{z} \frac{d \omega_{z}}{d t}=M_{z} \\
& \frac{d \vartheta}{d t}=\omega_{z} \\
& \vartheta=\theta+\alpha \\
& X=\frac{1}{2} \rho V^{2} S C_{D} \\
& Y=\frac{1}{2} \rho V^{2} S C_{L} \\
& M_{z}=\frac{1}{2} \rho V^{2} S L C_{m z}
\end{aligned}
$$


where $\rho$ is the atmospheric density, $S$ is the reference area, and $L$ is the reference length The aerodynamic model of the longitudinal channel drag coefficient $C_{D}$, lift coefficient $C_{L}$, and pitching moment coefficient $C_{m z}$ on angle of attack, angle of pitch-rudder deflection, and Mach number is:

$$
\left\{\begin{array}{l}
\rho=\rho_{0} \exp \left(\frac{-\left(H-H_{0}\right)}{H_{s}}\right) \\
C_{D}=C_{D}^{0}+C_{D}^{\alpha} \alpha+C_{D}^{\delta_{e}} \delta_{e}+C_{D}^{M a} M a+C_{D}^{M a \cdot \alpha} M a \cdot \alpha+C_{D}^{M a \cdot \delta_{e}} M a \cdot \delta_{e}+C_{D}^{M a^{2}} M a^{2} \\
C_{L}=C_{L}^{0}+C_{L}^{\alpha} \alpha+C_{L}^{\delta_{e}} \delta_{e}+C_{L}^{M a} M a+C_{L}^{\alpha^{2}} \alpha^{2}+C_{L}^{\alpha \cdot \delta_{e}} \alpha \cdot \delta_{e}+C_{L}^{M a \cdot \alpha} M a \cdot \alpha+C_{L}^{M a \cdot \delta_{e}} M a \cdot \delta_{e} \\
C_{m z}=C_{m z}^{0}+C_{m z}^{\alpha} \alpha+C_{m z}^{\delta_{e}} \delta_{e}+C_{m z}^{\alpha^{2}} \alpha^{2}+C_{m z}^{\alpha \cdot \delta_{e}} \alpha \cdot \delta_{e}+C_{m z}^{M a \cdot \alpha} M a \cdot \alpha+C_{m z}^{M a \cdot \delta_{e}} M a \cdot \delta_{e}
\end{array}\right.
$$

where $M a$ is the Mach number, $\rho_{0}$ is the standard atmospheric density, $H_{0}$ is the corresponding height, $1 / H_{s}$ is the attenuation law of atmospheric density, and $\delta_{e}$ is the elevator deflection angle. $C_{D}, C_{L}$, and $C_{m z}$ are the aerodynamic coefficients related to the angle of attack, angle of elevator, and Mach number, respectively.

By linearizing the above equation with a small disturbance, the longitudinal dynamic model of a hypersonic vehicle can be obtained as follows:

$$
\begin{aligned}
& \dot{x}=A x+B u \\
& y=C x+D u
\end{aligned}
$$

where $x=\left[\begin{array}{cc}\alpha & w_{z}\end{array}\right]^{T}$ and

$$
A=\left[\begin{array}{cc}
-a_{4} & 1 \\
-a_{2} & -a_{1}
\end{array}\right], B=\left[\begin{array}{l}
-a_{5} \\
-a_{3}
\end{array}\right], C=\left[\begin{array}{ll}
1 & 0 \\
0 & 1
\end{array}\right], D=\left[\begin{array}{l}
0 \\
0
\end{array}\right]
$$

We can then derive a hypersonic vehicle with a fairing as subsystem 1 :

$$
\begin{aligned}
& \dot{x}_{1}=A_{1} x+B_{1} u \\
& y_{1}=C_{1} x+D_{1} u
\end{aligned}
$$

A hypersonic vehicle without a fairing is subsystem 2:

$$
\begin{aligned}
& \dot{x}_{2}=A_{2} x+B_{2} u \\
& y_{2}=C_{2} x+D_{2} u
\end{aligned}
$$

where $A_{1}, B_{1}$ is the coefficient matrix of the hypersonic vehicle with the fairing, and $A_{2}, B_{2}$ is the coefficient matrix of that without the fairing.

\subsection{Design of Switching Controller for Hypersonic-Vehicle Fairing Separation}

The process of hypersonic-vehicle fairing separation can be regarded as switching from the subsystem with the fairing to the subsystem without the fairing. The control system is designed to find a controller that can realize arbitrary switching.

For the existence of an arbitrarily switched controller:

Assumption 1. There is a linear time-invariant system with state controllability:

$$
\dot{x}=A_{j} x_{(t)}+B_{j} u_{(t)}
$$

where $A_{j}$ and $B_{j}$ are the state matrix and input matrix of the jth subsystem, respectively.

Theorem 1. If there are symmetric positive definite matrices $W$ and $Z_{j}$, then: $A_{j} W+W A_{j}^{T}+B_{j} Z_{j}+Z_{j}^{T} B_{j}^{T}<0$ holds.

The state feedback controller is $u_{(t)}=K_{j} x_{(t)}$.

Among them, $K_{j}=Z_{j} W^{-1}$, and the closed-loop system is stable under any switching strategy. 
Proof of Theorem 1. The stability of the closed-loop system can be realized by the existence of the general Lyapunov function, i.e., $V_{(x)}=x^{T} P x$, let $P=W^{-1}$.

$$
\dot{x}=\left(A_{j}+B_{j} K_{j}\right) x_{(t)}
$$

Then:

$$
\begin{aligned}
& \dot{V}=\dot{x}_{(t)}^{T} P x_{(t)}+x_{(t)}^{T} P \dot{x}_{(t)}=x_{(t)}^{T}\left(A_{j}+B_{j} K_{j}\right)^{T} P x_{(t)}+x_{(t)}^{T} P\left(A_{j}+B_{j} K_{j}\right) x_{(t)} \\
& =x_{(t)}^{T}\left(A_{j}^{T}+K_{j}^{T} B_{j}^{T}\right) P x_{(t)}+x_{(t)}^{T} P\left(A_{j}+B_{j} K_{j}\right) x_{(t)} \\
& =x_{(t)}^{T} P\left(P^{-1} A_{j}^{T}+P^{-1} K_{j}^{T} B_{j}^{T}+A_{j} P^{-1}+B_{j} K_{j} P^{-1}\right) P x_{(t)} \\
& =x_{(t)}^{T} P\left(W A_{j}^{T}+W K_{j}^{T} B_{j}^{T}+A_{j} W+B_{j} K_{j} W\right) P x_{(t)} \\
& =x_{(t)}^{T} P\left(W A_{j}^{T}+A_{j} W+B_{j} Z_{j}+Z_{j}^{T} B_{j}^{T}\right) P x_{(t)}<0
\end{aligned}
$$

The system is stable; therefore, the proof is complete.

Assign the pole of $\dot{x}=\left(A_{j}+B_{j} K_{j}\right) x_{(t)}$ in a circle with center $\left(-d_{j}-r_{j}, 0\right)$ and radius $r_{j}$, which can be expressed as $C\left(d_{j}, r_{j}\right)$. Thus, the pole assignment of $L_{j}=A_{j}+B_{j} K_{j}$ is inside $C\left(d_{j}, r_{j}\right)$, as shown in Figure 7 .

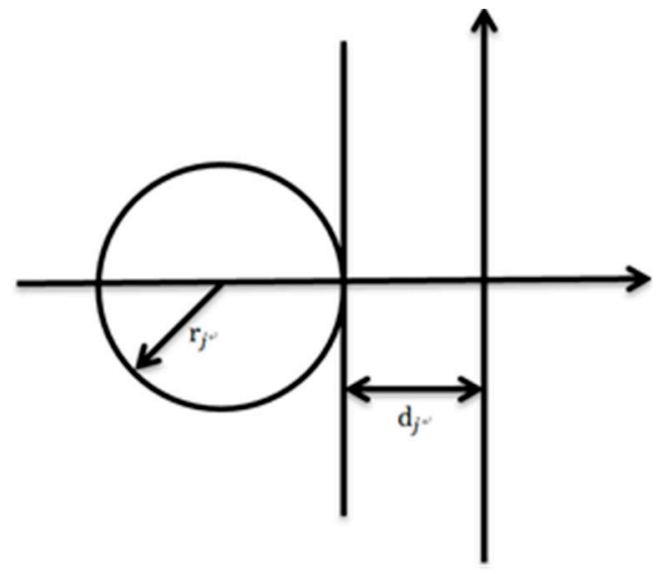

Figure 7. Schematic diagram of pole assignment of closed-loop system.

Theorem 2. If there are symmetric positive definite matrices $W$ and $Z_{j}$, then:

$$
\left[\begin{array}{cc}
A_{j} W+W A_{j}^{T}+B_{j} Z_{j}+Z_{j}^{T} B_{j}^{T}+2 d_{j} W & A_{j} W+B_{j} Z_{j}+d_{j} W \\
W A_{j}^{T}+Z_{j}^{T} B_{j}^{T}+d_{j} W & -r_{j} W
\end{array}\right]<0
$$

The pole of the system can be assigned in $C\left(d_{j}, r_{j}\right)$ and the system remains stable.

Proof of Theorem 2. The above formula can be expanded as

$$
\left[\begin{array}{cc}
A_{j} W+W A_{j}^{T}+B_{j} K_{j} W+W K_{j}^{T} B_{j}^{T}+2 d_{j} W & A_{j} W+B_{j} K_{j} W+d_{j} W \\
W A_{j}^{T}+W K_{j}^{T} B_{j}^{T}+d_{j} W & -r_{j} W
\end{array}\right]<0
$$

Based on Schur, we can derive that

$$
\begin{aligned}
& \left(L_{j}+d_{j} I\right) W+W\left(L_{j}+d_{j} I\right)^{T}+\frac{1}{r_{j}}\left(L_{j}+d_{j} I\right) W\left(L_{j}+d_{j} I\right)^{T} \\
& =A_{j} W+W A_{j}^{T}+B_{j} K_{j} W+W K_{j}^{T} B_{j}^{T}+2 d_{j} W \\
& +\frac{1}{r_{j}}\left(A_{j} W+B_{j} K_{j} W+d_{j} W\right) W^{-1}\left(W A_{j}^{T}+W K_{j}^{T} B_{j}^{T}+d_{j} W\right)<0
\end{aligned}
$$


It is suggested in Ref. [1] that the above conditions achieve pole placement and the system is stable when

$$
\begin{aligned}
& A_{j} W+W A_{j}^{T}+B_{j} K_{j} W+W K_{j}^{T} B_{j}^{T} \\
& <-2 d_{j} W-\frac{1}{r_{j}}\left(A_{j} W+B_{j} K_{j} W+d_{j} W\right) W^{-1}\left(W A_{j}^{T}+W K_{j}^{T} B_{j}^{T}+d_{j} W\right)<0
\end{aligned}
$$

2.4. Design of Switching Controller Considering Aerodynamic Interference in the Fairing-Separation Process

Assumption 2. The uncertainty term can be decomposed into the forms $\Delta A_{j}=D_{j} F_{j} E_{j}$ and $F_{j}^{T} F_{j} \leq I$, where $D_{j}$ and $E_{j}$ are constant matrices of appropriate dimensions and $F_{j}$ is the unknown matrix of appropriate dimensions.

Lemma 1. For any matrix $X, Y$ and real number $a>0$ with appropriate dimensions,

$$
X^{T} Y+Y^{T} X \leq a X^{T} X+\frac{1}{a} Y^{T} Y
$$

Let the switching system $\dot{x}_{(t)}=\left(A_{j}+\Delta A_{j}\right) x_{(t)}+B_{j} x_{(t)}$.

Theorem 3. If there are symmetric positive definite matrices $W$ and $Z_{j}$ and a real member $a>0$ that makes $\left[\begin{array}{cc}A_{j} W+W A_{j}^{T}+B_{j} Z_{j}+Z_{j}^{T} B_{j}^{T}+a D_{j} D_{j}^{T} & W E_{j}^{T} \\ E_{j} W & -a I\end{array}\right]<0$, the state feedback controller $u_{(t)}=K_{j} x_{(t)}$, where $K_{j}=Z_{j} W^{-1}$ and the closed-loop system is stable under any switching strategy.

Proof of Theorem 3. From the matrix inequality, it can be obtained that

$$
A_{j} W+W A_{j}^{T}+B_{j} Z_{j}+Z_{j}^{T} B_{j}^{T}+a D_{j} D_{j}^{T}+a^{-1} W E_{j}^{T} E_{j} W<0
$$

The closed-loop system can be achieved with the existing general Lyapunov function, i.e., $V_{(x)}=x^{T} P x$, and letting $P=W^{-1}$.

$$
\dot{x}=\left(A_{j}+\Delta A_{j}+B_{j} K_{j}\right) x_{(t)}
$$

$$
\begin{aligned}
& \dot{V}=\dot{x}_{(t)}^{T} P x_{(t)}+x_{(t)}^{T} P x_{(t)} \\
& =x_{(t)}^{T}\left(A_{j}+\Delta A_{j}+B_{j} K_{j}\right)^{T} P x_{(t)}+x_{(t)}^{T} P\left(A_{j}+\Delta A_{j}+B_{j} K_{j}\right) x_{(t)} \\
& =x_{(t)}^{T}\left(A_{j}^{T} P+P A_{j}+E_{j}^{T} F_{j}^{T} D_{j}^{T} P+P D_{j} F_{j} E_{j}+K_{j}^{T} B_{j}^{T} P+P B_{j} K_{j}\right) x_{(t)} \\
& =x^{T} P\left(W A_{j}^{T}+A_{j} W+W E_{j}^{T} F_{j}^{T} D_{j}^{T}+D_{j} E_{j} F_{j} W+B_{j} Z_{j}+Z_{j}^{T} B_{j}^{T}\right) P x
\end{aligned}
$$

It can be obtained from Lemma 1 that

$$
D_{j} F_{j} E_{j} W+W E_{j}^{T} F_{j}^{T} D_{j}^{T} \leq a D_{j} D_{j}^{T}+a^{-1} W E_{j}^{T} E_{j} W
$$

Additionally, $F_{j} F_{j}^{T} \leq I$, so

$$
\dot{V} \leq x^{T} P\left(W A_{j}^{T}+A_{j} W+Z_{j}^{T} B_{j}^{T}+B_{j} Z+a D_{j} D_{j}^{T}+a^{-1} W E_{j}^{T} E_{j} W\right) P x<0
$$

The proof is complete.

Assign the pole of the system $\dot{x}_{(t)}=\left(A_{j}+\Delta A_{j}\right) x_{(t)}+B_{j} x_{(t)}$ in a circle with center $C\left(\begin{array}{lll}-d_{j}-r_{j}, 0\end{array}\right)$ and radius $r_{j}$, which can be expressed as $C\left(d_{j} r_{j}\right)$, i.e., $\bar{A}_{j}=A_{j}+$ $\Delta A_{j}+B_{j} K_{j}$ is assigned inside $C\left(\begin{array}{ll}d_{j} & r_{j}\end{array}\right)$. 
Theorem 4. If there are symmetric positive definite matrices $W$ and matrix $Z_{j}$ and real member $a>0$, then

$$
\left[\begin{array}{cccc}
A_{j} W+W A_{j}^{T}+B_{j} Z_{j}+Z_{j}^{T} B_{j}^{T}+a D_{j} D_{j}^{T}+2 d_{j} W & W E_{j}^{T} & A_{j} W+B_{j} Z_{j}+d_{j} W & 0 \\
E_{j} W & -a I & 0 & 0 \\
W A_{j}^{T}+Z_{j}^{T} B_{j}^{T}+d_{j} W & 0 & -r_{j} W & W E_{j}^{T} \\
0 & 0 & E_{j} W & -a I
\end{array}\right]<0
$$

The pole of the closed-loop system can be assigned in $C\left(\begin{array}{ll}d_{j} & r_{j}\end{array}\right)$ and the system is stable.

Proof of Theorem 4. Let

$$
L=A_{j} W+W A_{j}^{T}+B_{j} Z_{j}+Z_{j}^{T} B_{j}^{T}=A_{j} W+W A_{j}^{T}+B_{j} K_{j} W+W K_{j}^{T} B_{j}^{T}
$$

From the above matrix inequality, we can derive

$$
\left[\begin{array}{cccc}
L+a D_{j} D_{j}^{T}+2 d_{j} W & W E_{j}^{T} & A_{j} W+B_{j} K_{j} W+d_{j} W & 0 \\
E_{j} W & -a I & 0 & 0 \\
W A_{j}^{T}+W K_{j}^{T} B_{j}^{T}+d_{j} W & 0 & -r_{j} W & W E_{j}^{T} \\
0 & 0 & E_{j} W & -a I
\end{array}\right]<0
$$

The above formula is equivalent to

$$
\left[\begin{array}{cc}
L+2 a D_{j} D_{j}^{T}+2 d_{j} W+a^{-1} W E_{j}^{T} E_{j}^{T} W & A_{j} W+B_{j} K_{j} W+d_{j} W \\
W A_{j}^{T}+W K_{j}^{T} B_{j}^{T}+d_{j} W & -r_{j} W+W E_{j}^{T} E_{j} W
\end{array}\right]<0
$$

Define matrix

$$
Y_{j}=\left[\begin{array}{cc}
L+a D_{j} D_{j}^{T}+2 d_{j} W+a^{-1} W E_{j}^{T} E_{j}^{T} W & A_{j} W+B_{j} K_{j} W+d_{j} W \\
W A_{j}^{T}+W K_{j}^{T} B_{j}^{T}+d_{j} W & -r_{j} W
\end{array}\right]
$$

Then,

$$
\left[\begin{array}{cc}
L+2 a D_{j} D_{j}^{T}+2 d_{j} W+a^{-1} W E_{j}^{T} E_{j}^{T} W & A_{j} W+B_{j} K_{j} W+d_{j} W \\
W A_{j}^{T}+W K_{j}^{T} B_{j}^{T}+d_{j} W & -r_{j} W+W E_{j}^{T} E_{j} W
\end{array}\right]<0
$$

which is equivalent to

$$
Y_{j}+\left[\begin{array}{cc}
D_{j} & 0 \\
0 & 0
\end{array}\right]\left[\begin{array}{cc}
D_{j}^{T} & 0 \\
0 & 0
\end{array}\right]+a^{-1}\left[\begin{array}{cc}
0 & 0 \\
W E_{j}^{T} & 0
\end{array}\right]\left[\begin{array}{cc}
0 & E_{j} W \\
0 & 0
\end{array}\right]<0
$$

This obtains

$$
Y_{j}+a\left[\begin{array}{cc}
D_{j} & 0 \\
0 & 0
\end{array}\right]\left[\begin{array}{cc}
D_{j}^{T} & 0 \\
0 & 0
\end{array}\right]+a^{-1}\left[\begin{array}{cc}
0 & 0 \\
W E_{j}^{T} & 0
\end{array}\right] F_{j}^{T} F_{j}\left[\begin{array}{cc}
0 & E_{j} W \\
0 & 0
\end{array}\right]<0
$$

From Lemma 1:

$$
Y_{j}+a\left[\begin{array}{cc}
D_{j} & 0 \\
0 & 0
\end{array}\right] F_{j}\left[\begin{array}{cc}
0 & E_{j} W \\
0 & 0
\end{array}\right]+\left[\begin{array}{cc}
0 & 0 \\
W E_{j}^{T} & 0
\end{array}\right] F_{j}^{T}\left[\begin{array}{cc}
D_{j}^{T} & 0 \\
0 & 0
\end{array}\right]<0
$$

which can be expanded as

$$
\left[\begin{array}{cc}
L+a D_{j} D_{j}^{T}+2 d_{j} W+a^{-1} W E_{j}^{T} E_{j}^{T} W & A_{j} W+B_{j} K_{j} W+d_{j} W+D_{j} F_{j} E_{j} W \\
W A_{j}^{T}+W K_{j}^{T} B_{j}^{T}+d_{j} W+W E_{j}^{T} F_{j}^{T} D_{j}^{T} & -r_{j} W
\end{array}\right]<0
$$


Thus,

$L+a D_{j} D_{j}^{T}+2 d_{j} W+a^{-1} W E_{j}^{T} E_{j}^{T} W$
$+\frac{1}{r_{j}}\left(A_{j} W+B_{j} K_{j} W+d_{j} W+D_{j} F_{j} E_{j} W\right) W^{-1}\left(W A_{j}^{T}+W K_{j}^{T} B_{j}^{T}+d_{j} W+W E_{j}^{T} F_{j}^{T} D_{j}^{T}\right)$
$<0$

Let $\bar{A}_{j}=A_{j}+\Delta A_{j}+B_{j} K_{j}$ and

$$
\begin{aligned}
& \left(\bar{A}_{j}+d_{j} I\right) W+W\left(\bar{A}_{j}+d_{j} I\right)^{T}+\frac{1}{r_{j}}\left(\bar{A}_{j}+d_{j} I\right) W\left(\bar{A}_{j}+d_{j} I\right)^{T} \\
& =L+D_{j} F_{j} E_{j} W+W E_{j}^{T} F_{j}^{T} D_{j}^{T}+2 d_{j} W \\
& +\frac{1}{r_{j}}\left(A_{j} W+B_{j} K_{j} W+d_{j} W+D_{j} F_{j} E_{j} W\right) W^{-1}\left(W A_{j}^{T}+W K_{j}^{T} B_{j}^{T}+d_{j} W+W E_{j}^{T} F_{j}^{T} D_{j}^{T}\right)
\end{aligned}
$$

Then, we can derive from Lemma 1 that

$$
\begin{aligned}
& L+D_{j} F_{j} E_{j} W+W E_{j}^{T} F_{j}^{T} D_{j}^{T}+2 d_{j} W+\frac{1}{r_{j}}\left(A_{j} W+B_{j} K_{j} W+d_{j} W\right. \\
& \left.+D_{j} F_{j} E_{j} W\right) W^{-1}\left(W A_{j}^{T}+W K_{j}^{T} B_{j}^{T}+d_{j} W+W E_{j}^{T} F_{j}^{T} D_{j}^{T}\right) \\
& <L+a D_{j} D_{j}^{T}+2 d_{j} W+a^{-1} W E_{j}^{T} E_{j}^{T} W+\frac{1}{r_{j}}\left(A_{j} W+B_{j} K_{j} W\right. \\
& \left.+d_{j} W+D_{j} F_{j} E_{j} W\right) W^{-1}\left(W A_{j}^{T}+W K_{j}^{T} B_{j}^{T}+d_{j} W+W E_{j}^{T} F_{j}^{T} D_{j}^{T}\right)<0
\end{aligned}
$$

which is

$$
\left(\bar{A}_{j}+d_{j} I\right) W+W\left(\bar{A}_{j}+d_{j} I\right)^{T}+\frac{1}{r_{j}}\left(\bar{A}_{j}+d_{j} I\right) W\left(\bar{A}_{j}+d_{j} I\right)^{T}<0
$$

According to Ref. [1], the above conditions achieve pole assignment and the formula is

$$
\begin{aligned}
& L+D_{j} F_{j} E_{j} W+W E_{j}^{T} F_{j}^{T} D_{j}^{T}<-2 d_{j} W-\frac{1}{r_{j}}\left(A_{j} W+B_{j} K_{j} W+d_{j} W\right. \\
& \left.+D_{j} F_{j} E_{j} W\right) W^{-1}\left(W A_{j}^{T}+W K_{j}^{T} B_{j}^{T}+d_{j} W+W E_{j}^{T} F_{j}^{T} D_{j}^{T}\right)<0
\end{aligned}
$$

which keeps the system stable.

\section{Results}

\subsection{The Simulation Verification for LMI}

We assume that the hypersonic vehicle flew at an altitude of $21 \mathrm{~km}$ with a flying speed of 5.6 Ma and activated the fairing separation after the $20 \mathrm{~s}$ boosting separation. When the fairing was separating, the initial angle of attack $\alpha=0^{\circ}$, and the initial angular velocity $w_{z}=1^{\circ} / \mathrm{s}$. The system before and after the fairing separation were denoted as subsystem 1 and subsystem 2, respectively, switching from subsystem 1 to subsystem 2 at $20 \mathrm{~s}$. The dynamic system matrix before and after the fairing separation could be calculated as below:

$$
\begin{aligned}
& A_{1}=\left[\begin{array}{cc}
-0.3563 & 23.7567 \\
1 & -0.0955
\end{array}\right], A_{2}=\left[\begin{array}{cc}
-0.3632 & 9.2644 \\
1 & -0.2039
\end{array}\right] \\
& B_{1}=\left[\begin{array}{c}
-25.4341 \\
0
\end{array}\right], B_{2}=\left[\begin{array}{c}
-74.0901 \\
-0.0355
\end{array}\right]
\end{aligned}
$$

Using Theorem 1, comparing Figures 8 and 9, it can be seen that the switching system obtained an asymptotically stable general Lyapunov on the state feedback control, and better state responses of pitching angular velocity and power angle are obtained with the pole assignment. 


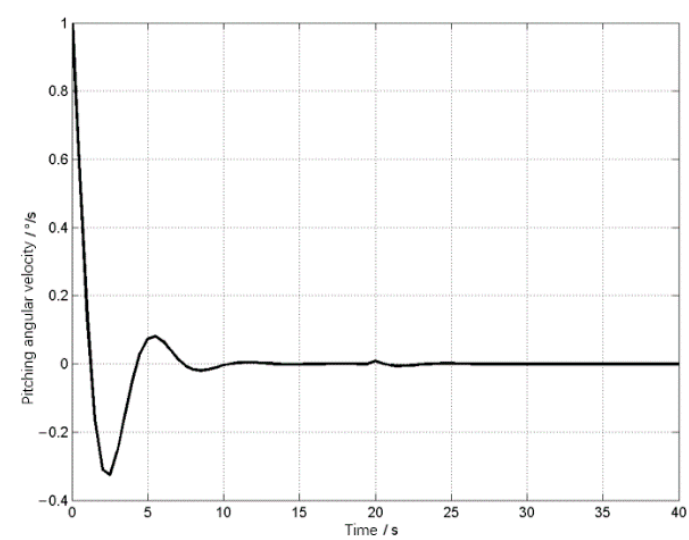

(a)

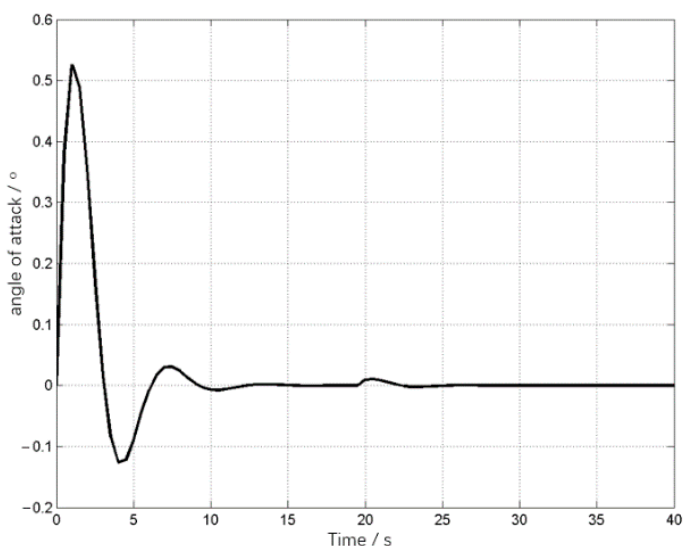

(b)

Figure 8. (a)State-response graph of the pitching angular velocity; (b) state-response graph of the angle of attack.

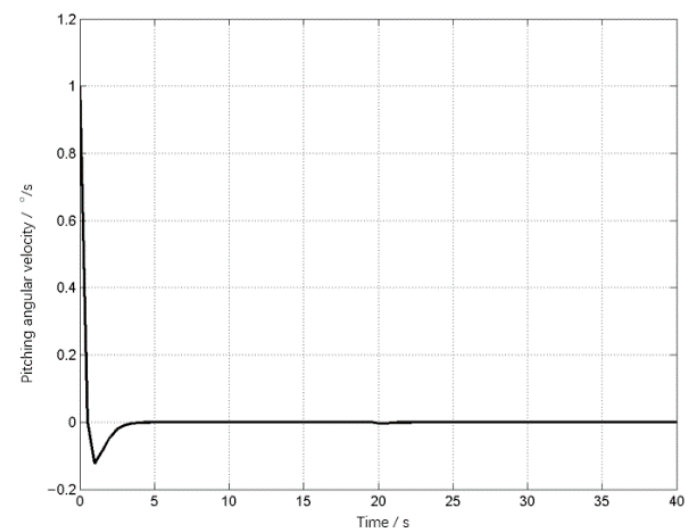

(a)

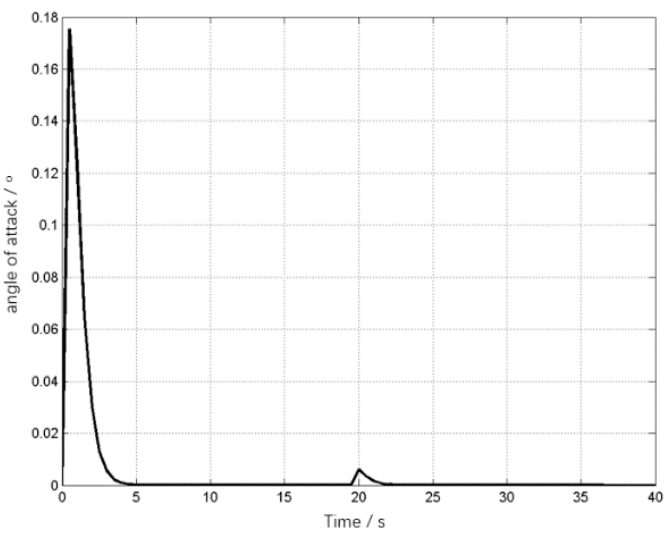

(b)

Figure 9. (a) State-response graph of the pitching angular velocity with pole assigned; (b) stateresponse graph of the angle of attack with pole assigned.

\subsection{The Simulation Verification Considering Aerodynamic Interference}

To verify the effectiveness of the fairing separation and interference suppression of the proposed switching-based control method, numerical simulations on the separation motion of the hypersonic vehicle were conducted. The experimental environment was established with a 4-core, 64-bit, 2.4 GHz, 8 GB RAM Intel i7 computer and MATLAB R2013a software.

It was assumed that the hypersonic vehicle flew at an altitude of $21 \mathrm{~km}$ with a flying speed of 5.6 Ma and activated the fairing separation after the $20 \mathrm{~s}$ boosting separation. When the fairing was separated, the initial angle of attack $\alpha=0^{\circ}$, and the initial angular velocity $w_{z}=1^{\circ} / \mathrm{s}$, and the interference generated during the fairing separation is given in the form of uncertainty. The systems before and after the fairing separation are subsystem 
1 and subsystem 2, respectively, switching from subsystem 1 to subsystem 2 at $20 \mathrm{~s}$. Its dynamic system is as below:

$$
\begin{aligned}
& A_{1}=\left[\begin{array}{cc}
-0.3563 & -23.7567 \\
1 & -0.0955
\end{array}\right], A_{2}=\left[\begin{array}{cc}
-0.3632 & -9.2644 \\
1 & -0.2039
\end{array}\right] \\
& B_{1}=\left[\begin{array}{c}
-25.4341 \\
0
\end{array}\right], B_{2}=\left[\begin{array}{c}
-74.0901 \\
-0.0355
\end{array}\right] \\
& \Delta A_{1}=\left[\begin{array}{cc}
0 & 2.5 \\
0 & 0.01
\end{array}\right], \Delta A_{2}=\left[\begin{array}{cc}
0 & 2 \\
0 & 0.009
\end{array}\right] \\
& D_{1}=\left[\begin{array}{c}
2.5 \\
0.01
\end{array}\right], D_{2}=\left[\begin{array}{c}
2 \\
0.009
\end{array}\right] \\
& F_{1}=F_{2}=I \\
& E_{1}=E_{2}=\left[\begin{array}{ll}
0 & 1
\end{array}\right]
\end{aligned}
$$

The state-response graphs of pitching angular velocity and angle of attack are shown in Figure 10. In Figure 11, the blue curve represents the changing trend in the pitching moment coefficient of the aircraft during fairing separation in the actual flight test, and the black curve represents the changing trend in the pitching moment coefficient during the fairing separation in the simulation test.

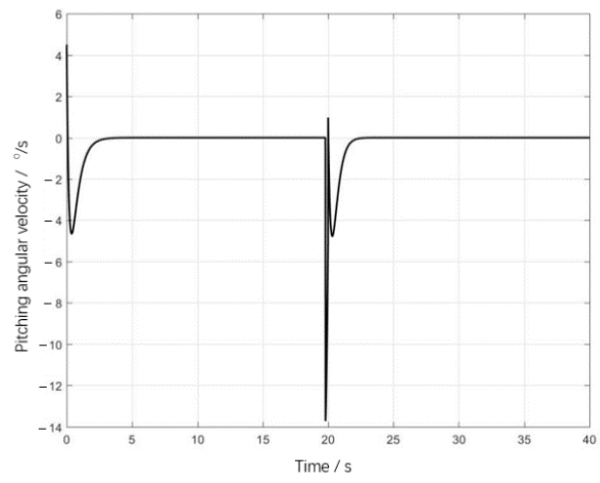

(a)

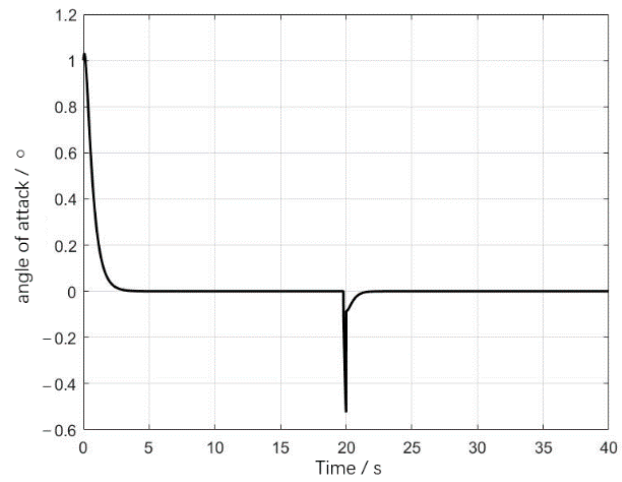

(b)

Figure 10. (a) State-response graph of the pitching angular velocity; (b) state-response graph of the power angle.

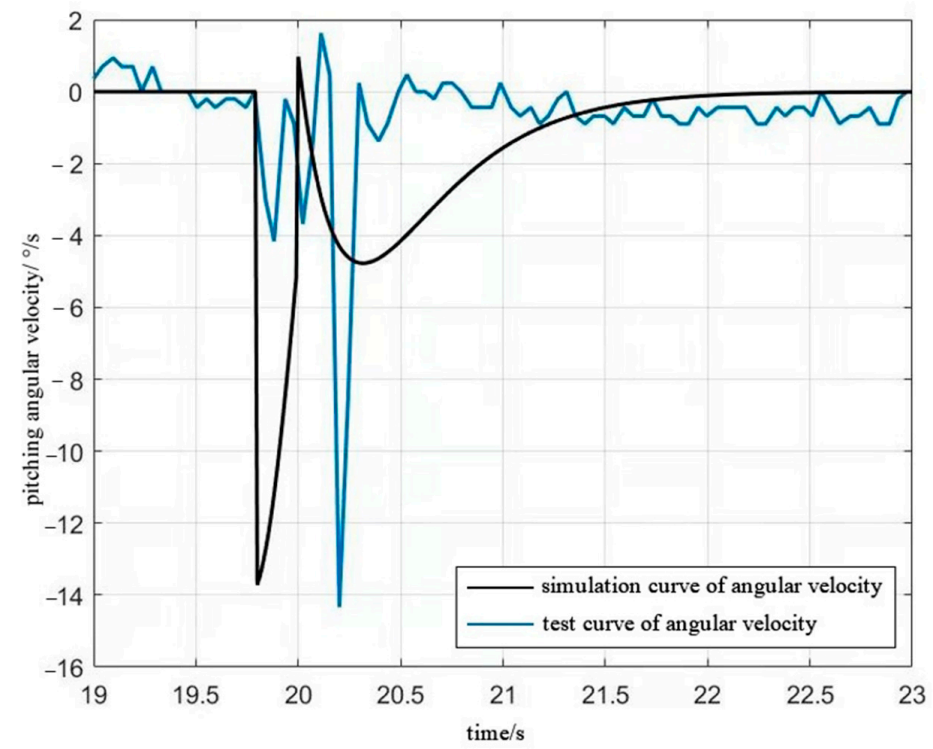

Figure 11. Comparison diagram of the simulation curve and test curve of angular velocity. 


\section{Discussion}

It can be seen from Figure 8 that the linear steady-switching system obtained an effect of asymptotic Lyapunov stability. However, because the pitching angular velocity and angle of attack change too much at the moment of switching, the dynamic characteristics of the aircraft would be unacceptable in actual flight.

Based on the above problem, the pole of the switching system was assigned in a circle of $C(2,0.5)$ by applying Theorem 2 . The state-response graphs of pitching angular velocity and angle of attack are shown in Figure 9a,b, respectively.

As Figure 9 shows, the switching system after pole assignment exhibited a small change in the pitch angular velocity and the angle of attack at the moment of switching, and Lyapunov was asymptotically stable.

The simulation Figure 10 in Section 3.2 shows that when considering the aerodynamic interference generated by the fairing, switching control could not only realize reliable separation of the fairing for hypersonic aircraft under high dynamic pressure, but also quickly suppressed the interference of the separation process with the attitude and flight state of the aircraft.

Figure 11 shows a partially enlarged image of the fairing separation and a comparison of the simulation curve with the test curve. It can be seen that the simulation curve is very close to the experimental results. In the fairing-separation process, the interference of external airflow with the spacecraft attitude has an angular velocity of about $14^{\circ} / \mathrm{s}$, and the switching control can achieve rapid suppression of the aerodynamic interference.

The simulation shows that when considering the aerodynamic interference generated by the fairing, switching control can not only realize the reliable separation of the fairing for hypersonic aircraft under high dynamic pressure, but also quickly suppress the interference of the separation process with the attitude and flight state of the aircraft.

\section{Conclusions}

Aiming to suppress interference for hypersonic vehicles undergoing fairing separation under high dynamic pressure, this paper proposes a method of switching-based control that integrates the two states of the aircraft with and without fairing into a switchable subsystem. Based on the linear matrix inequality (LMI) approach, the attitude stability in fairing separation is converted into arbitrary switching of the switching system. Meanwhile, considering the aerodynamic interaction of the fairing movement with the aircraft during the separation, a control system for suppressing interference during fairing separation has been designed. Simulation results show that the designed control system can realize reliable separation of the fairing under high dynamic pressure and achieve effective suppression of the interference in the separation process, with the advantages of stability and rapidity.

Author Contributions: Conceptualization, Y.F. and Q.Z.; methodology, Y.F.; software, W.W.; validation, Q.Z. and Y.F.; investigation, Q.Z.; resources, Y.F.; data curation, Q.Z.; writing-original draft preparation, Q.Z.; writing-review and editing, Y.F.; visualization, Q.Z.; supervision, Y.F. and W.W.; project administration, Y.F. and W.W. All authors have read and agreed to the published version of the manuscript.

Funding: This research received no external funding.

Conflicts of Interest: The authors declare no conflict of interest.

\section{References}

1. Liever, P.A.; Habchi, S.D.; Engelund, W.C.; Martin, J.G. Stage Separation Analysis of the X-43A Research Vehicle. In Proceedings of the 22nd Applied Aerodynamics Conference and Exhibit, Providence, RI, USA, 16-19 August 2004; AIAA $2004-4725$.

2. Blocker, W.D.; Ruebush, D.E. X-43A Stage Separation System-A Flight Data Evaluation. In Proceedings of the AIAA/CIRA 13th International Space Planes and Hypersonics Systems and Technologies Conference, Capua, Italy, 16-20 May 2005; AIAA 2005-3335.

3. Buning, P.G.; Wong, T.C.; Dilley, A.D.; Pao, J.L. Computational fluid dynamics prediction of Hyper-X stage separation aerodynamics. J. Spacecr. Rockets 2001, 38, 820-827. [CrossRef] 
4. Woods, W.C.; Holland, S.D.; DiFulvio, M. Hyper-X stage separation wind-tunnel test program. J. Spacecr. Rockets 2001, 38, 811-819. [CrossRef]

5. Tartabini, P.V.; Bose, D.M.; McMinn, J.D.; Martin, J.G.; Strovers, B.K. Hyper-X stage separation trajectory validation studies. In Proceedings of the AIAA Modeling and Simulation Technologies Conference and Exhibit, Austin, TX, USA, 11-14 August 2003; AIAA 2003-5819.

6. Shengjiang, Y.; Chaoyi, L. Research on Interstage Separation Control Technology of Hypersonic Aircraft in the United States. Flight Missile 2014, 11, 34-42.

7. Yongtao, L.; Xiangju, Q.; Wenqian, T. Dynamics Modeling and Simulation of Air-breathing Hypersonic Flight Aircraft. Flight Mech. 2014, 32, 403-407.

8. Yanshuo, Y. Pole Assignment of Uncertain Switching System Based on LMI. Master's Thesis, Xiamen University, Xiamen, China, 2008.

9. Margaliot, M. Stability analysis of switched systems using variational principles: An introduction. Automatica 2006, 42, 2059-2077. [CrossRef]

10. Dogruel, M.; Ozguner, U. Stability of a set of matrices: An application to hybrid systems. In Proceedings of the Tenth International Symposium on Intelligent Control, Monterey, CA, USA, 27-29 August 1995.

11. Jianqiang, C.; Xiaojun, W.; Jian, Z.; Bin, L.; Hongyin, J.; Naichun, Z. Nonstructural general CFD software for national numerical wind tunnel (NNW) project. J. Aeronaut. 2021, 42, 9-30, 32.

12. Zhang, P.; Jia, H.; Guo, Y.; Zhou, G.; Wu, X.; Zhao, W. Simulation of aerodynamic characteristics of grid rudder based on flowstar software. J. Comput. Mech. 2022. Available online: https://kns.cnki.net/kcms/detail/detail.aspx?FileName=JSJG20210701014 $\& D b N a m e=C A P J 2021$ (accessed on 13 February 2022). 